\title{
Dose per muscle in cervical dystonia: pooled data from seven movement disorder centres
}

\author{
Wolfgang H. Jost ${ }^{1}$, Artur Drużdż ${ }^{2}$ Sanjay Pandey ${ }^{3}$, Bo Biering-Sørensen ${ }^{4}$, Alexandre Kreisler ${ }^{5}$, \\ Laurent Tatu ${ }^{6}$, Christian F. Altmann ${ }^{1}$, Jarosław Sławek ${ }^{7,8}$ \\ ${ }^{1}$ Parkinson-Klinik Ortenau, Wolfach, Germany \\ ${ }^{2}$ Department of Neurology, Municipal Hospital in Poznan, Poznan, Poland \\ ${ }^{3}$ Department of Neurology, Govind Ballabh Pant Institute of Postgraduate \\ Medical Education and Research, JLN Marg, New Delhi, India \\ ${ }^{4}$ Movement Disorder Clinic, Department of Neurology, Rigshospitalet, Copenhagen, Denmark \\ ${ }^{5}$ Service de Neurologie et Pathologie du Mouvement, CHRU de Lille, France \\ ${ }^{6}$ Department of Neuromuscular Diseases and Department of Anatomy, CHRU Besançon, \\ University of Franche-Comté, Besançon, France \\ ${ }^{7}$ Division of Neurological and Psychiatric Nursing, Faculty of Health Sciences, Medical University of Gdansk, Poland; \\ ${ }^{8}$ Department of Neurology and Stroke, St. Adalbert Hospital, Gdansk, Poland
}

\begin{abstract}
Aim of the study. Botulinum neurotoxin type-A (BoNT/A) injections are the established treatment in cervical dystonia (CD). But clinical practice regarding the choice of muscles into which injections are made varies between centres. Until now, there have been no dose-per-muscle recommendations based on 'searching the dose' clinical trial data.

Clinical rationale for study. We therefore examined the dosages under real world conditions at seven international movement disorders centres, using an identical clinical approach.

Results. We examined 305 patients with CD (55.6 \pm 13.2 years, 204 female). The most commonly injected muscles were the splenius capitis (84.9\%), sternocleidomastoid (80.3\%), trapezius (59.7\%), levator scapulae (49.8\%), semispinalis capitis (39\%), and obliquus capitis inferior (36.7\%). The mean total dose per treatment session with aboBoNT/A was 652.5 (SD = 285.5), with onaBoNT/A it was 159.5 (SD = 62.4), and with incoBoNT/A it was 173.4 (SD = 99.2) units. The doses injected into each muscle in the ona- or incoBoNT/A groups were between 19.7 and 48.2 units, with the highest dose for the splenius capitis with 49.2 \pm 26.0 units. The doses in the aboBoNT/A group were between 69.6 and 146.4 units, and the highest dose being injected into the splenius capitis (139.6 \pm 80.7 units).

Conclusions and clinical implications. In clinical trials the doses per muscle are based on an arbitrary decision. In our study, the doses were lower than in other studies, which may be due to the number of muscles per session, the use of ultrasound guidance (and therefore more precise injections), as well as the use of the Col-Cap concept. Our results exemplify everyday practice, and may help as the basis for recommendations and further investigations.
\end{abstract}

Key words: torticollis, cervical dystonia, Col-Cap concept, botulinum toxin, sternocleidomastoideus muscle, splenius capitis muscle

(Neurol Neurochir Pol 2021; 55 (2): 174-178) 


\section{Introduction}

The choice of muscles for injections in cervical dystonia (CD) varies between centres, and is based both on different concepts and on personal experience. There are no generally accepted dose recommendations based on dose-finding studies. However, a few studies have shown the dose per muscle used in a randomised and open (observational) manner [1-3]. In the summary of products characteristics (SPC) of all botulinum neurotoxin-A products (BoNT/A) there are only indicated maximal recommended doses. For some products, specific muscles are mentioned but without specified dose recommendations (SPC Botox ${ }^{\circ}$, SPC Dysport ${ }^{\circ}$, SPC Xeomin ${ }^{\circ}$ ). Therefore, the standard dose recommendations are mostly based on pivotal clinical trials and personal experience $[4,5]$. The majority of studies in $\mathrm{CD}$ were performed many years ago. In 2009, a new concept (the Col-Cap) was introduced, expanding not only the number of $\mathrm{CD}$ patterns, but also the number of muscles to be injected $[6,7]$.

The aim of our study was to look for effective doses per muscle used in everyday clinical practice in different centres using a similar treatment regime. As a data basis for further studies, we therefore analysed pooled data on usually-chosen dosages per muscle in a larger collective study in previously injected patients with established muscle patterns.

\section{Materials and methods}

Between 1 January and 30 June 2019, we examined retrospectively in seven centres specialized in movement disorders, 311 consecutive patients with $\mathrm{CD}$, who were being already successfully treated (with a moderate or good response clinically determined by patient and physician) for at least three times. Consecutive patients were included if they had idiopathic $\mathrm{CD}$, with pronounced symptoms interfering with their daily activities, and who had been admitted at least three months after their previous BoNT/A treatment, the effect of which had worn off. Six patients were excluded from our study because of incomplete data, thus resulting in a sample size $\mathrm{n}=305$. The centres involved were: Besançon (France), Copenhagen (Denmark), Gdansk (Poland), Lille (France), New Delhi (India), Poznan (Poland), and Wolfach (Germany). All investigators were specialists in movement disorders with long-term experience with BoNT/A treatment in CD (at least 15 years each). All injections were performed by investigators trained in the use of ultrasonography guidance (US). The therapeutic approach (treatment regime) across all centres was uniform and based on the Col-Cap concept [6]. BoNT/A was diluted according to the SPC recommendations: ona- and incoBoNT/A vials containing 100 units were reconstituted with $2 \mathrm{ml}$, and aboBoNT/A vials with 300 and 500 units with $1.5 \mathrm{ml}$ and $2.5 \mathrm{ml}$ of $0.9 \% \mathrm{NaCl}$ respectively.

Patients were excluded if at least a moderate effect of previous BoNT/A injections had not been obtained and if the co-morbidities (e.g. severe depression) could influence the overall subjective assessment of the results. Concomitant use of neuroleptics was forbidden, and other causes of CD (suggesting symptomatic or pseudodystonic origin) were excluded.

\section{Results}

305 patients with $\mathrm{CD}$ (mean age $55.6 \pm 13.2$ years, range 21-90, 204 female) were injected and assessed.

The most common primary form of $\mathrm{CD}$ in our group was torticaput (49\%) and the second most common was laterocaput (16.7\%). All other subtypes afflicted less than $10 \%$ of our study population. Pure forms were observed in $16.3 \%$ of patients only. Torticaput was combined in $46 \%$ with laterocaput, and in $20.7 \%$ with retrocaput. Laterocaput was combined mainly with torticaput $(45.1 \%)$, laterocollis $(33.2 \%)$ or retrocaput (23.5\%). Shift forms were found in $14.7 \%$. On average, patients had $2.51( \pm$ SD 1.09) subtypes each, and tremor was observed in $55.6 \%[5,8]$.

The most commonly injected muscles were the splenius capitis $(84.9 \%)$, sternocleidomastoid $(80.3 \%)$, trapezius (59.7\%), levator scapulae (49.8\%), semispinalis capitis (39\%), and obliquus capitis inferior (36.7\%) respectively. 154 patients received onabotulinumtoxinA (onaBoNT/A), 53 patients incobotulinumtoxinA (incoBoNT/A), and 98 abobotulinumtoxin $\mathrm{A}$ (aboBoNT/A). The mean total dose for a treatment session with aboBoNT/A was $652.5(\mathrm{SD}=285.5)$ units, for ona- 159.5 (SD = 62.4) units, and for incoBoNT/A 173.4 (SD $=99.2)$ units respectively. The doses injected into each muscle are set out in Table 1. The doses injected into each muscle in the ona- or incoBoNT/A groups were between 19.7 and 49.2 units. The highest dose was injected into the splenius capitis, $49.2 \pm 26.0$ units, with the highest total dose per session being 130 units. The lowest doses were chosen for both semispinalis muscles.

The doses in the aboBoNT/A group were between 75.4 and 139.6 units (Tab. 1B). The highest dose was injected into the splenius capitis with $139.6 \pm 80.7$ units, with the highest dose of 400 units total per session. The lowest doses were chosen for semispinalis cervicis, longissimus (cervicis and/or capitis) and medial scalene muscles.

\section{Discussion}

Although BoNT/A injections are the therapy of choice for $\mathrm{CD}$, the dose per muscle is an unresolved problem $[7,9]$. Both total dose per session and per muscle were pre-established at study design in clinical trials and incorporated into published recommendations [1,2]. SPCs are mostly focused on maximal total dose (SPC Botox ${ }^{\circ}$, SPC Dysport ${ }^{\circ}$, SPC Xeomin ${ }^{\circ}$ ). Recently published versions of SPC do not include the specific doses per muscle, and only aboBoNT/A SPC recommends for head tilt 150 units to sternocleidomatoid muscle (SCM) and 350 units to splenius capitis (SC). 
Table 1A. Dose per muscle of abobotulinumtoxinA

\begin{tabular}{|c|c|c|c|c|c|c|c|c|c|c|}
\hline Muscle & SCM & SM & LS & SsCap & SsCer & sCap & sCer & OCl & Trap & Long \\
\hline Mean & 117.9 & 87.5 & 135.8 & 111.4 & 102.1 & 139.6 & 75.4 & 117.3 & 123.4 & 87.3 \\
\hline SD & 40.1 & 36.8 & 50.8 & 63.4 & 73.9 & 80.7 & 47.5 & 43.6 & 47.7 & 35.7 \\
\hline MAX & 200 & 175 & 250 & 380 & 400 & 400 & 200 & 200 & 250 & 160 \\
\hline MIN & 40 & 20 & 40 & 40 & 10 & 25 & 25 & 40 & 40 & 25 \\
\hline $\mathrm{N}$ & 77 & 18 & 59 & 45 & 28 & 82 & 11 & 44 & 74 & 15 \\
\hline Proportion & $78.6 \%$ & $18.4 \%$ & $60.2 \%$ & $45.9 \%$ & $28.6 \%$ & $83.7 \%$ & $11.2 \%$ & $44.9 \%$ & $75.5 \%$ & $15.3 \%$ \\
\hline
\end{tabular}

All others: Mean $=87.3 ; \mathrm{SD}=54.6 ; \mathrm{MAX}=240 ; \mathrm{MIN}=30 ; \mathrm{N}=13 ;$ Proportion $=13.3 \%$

Table 1B. Dose per muscle of inco-BoNT/A

\begin{tabular}{|c|c|c|c|c|c|c|c|c|c|c|}
\hline Muscle & SCM & SM & LS & SsCap & SsCer & SCap & sCer & OCI & Trap & Long \\
\hline Mean & 34.7 & 28.8 & 39.0 & 30.7 & 31.3 & 36.8 & 65.0 & 31.7 & 33.0 & 27.0 \\
\hline SD & 15.2 & 16.5 & 17.9 & 15.2 & 14.3 & 21.2 & 49.5 & 20.9 & 13.3 & 10.6 \\
\hline MAX & 70 & 50 & 80 & 70 & 50 & 100 & 100 & 90 & 60 & 40 \\
\hline MIN & 15 & 10 & 15 & 10 & 10 & 10 & 30 & 5 & 10 & 10 \\
\hline $\mathrm{N}$ & 43 & 4 & 34 & 15 & 8 & 43 & 2 & 23 & 35 & 10 \\
\hline Proportion & $81.1 \%$ & $7.6 \%$ & $64.2 \%$ & $28.3 \%$ & $15.1 \%$ & $81.1 \%$ & $3.8 \%$ & $43.4 \%$ & $66.0 \%$ & $18.9 \%$ \\
\hline
\end{tabular}

All others: Mean $=23.3 ; \mathrm{SD}=15.3 ; \mathrm{MAX}=40 ; \mathrm{MIN}=10 ; \mathrm{N}=3 ;$ Proportion $=5.7 \%$

Table 1C. Dose per muscle of ona-BoNT/A

\begin{tabular}{|c|c|c|c|c|c|c|c|c|c|c|}
\hline Muscle & SCM & SM & LS & SsCap & SsCer & scap & scer & OCI & Trap & Long \\
\hline Mean & 40.8 & 24.3 & 32.8 & 19.7 & 18.0 & 49.2 & 13.3 & 20.6 & 29.2 & 20.5 \\
\hline SD & 15.5 & 5.8 & 12.3 & 13.2 & 8.5 & 26.0 & 4.8 & 11.4 & 13.2 & 9.9 \\
\hline MAX & 80 & 30 & 70 & 100 & 40 & 130 & 20 & 50 & 100 & 40 \\
\hline MIN & 7.5 & 10 & 10 & 7.5 & 5 & 10 & 5 & 7.5 & 10 & 5 \\
\hline $\mathrm{N}$ & 125 & 14 & 59 & 59 & 37 & 134 & 9 & 45 & 73 & 27 \\
\hline Proportion & $81.2 \%$ & $9.1 \%$ & $38.3 \%$ & $38.3 \%$ & $24.0 \%$ & $87.0 \%$ & $5.8 \%$ & $29.2 \%$ & $47.4 \%$ & $17.5 \%$ \\
\hline
\end{tabular}

All other: Mean $=22.5 ; \mathrm{SD}=16.1 ; \mathrm{MAX}=90 ; \mathrm{MIN}=7.5 ; \mathrm{N}=47 ;$ Proportion $=30.5 \%$

SCM — sternocleidomastoideus; SM — scalenus muscles; LS — levator scapulae; SsCap — semispinalis capitis; SsCer — semispinalis cervicis; SCap — splenius capitis; SCer — splenius cervicis; OCI — obliquus capitis inferior; Trap — trapezius; Long - longissimus; $\mathrm{N}$ - number of muscles injected

Regarding only the main double-blind, placebo-controlled, randomised studies: in a majority of them the number of injection sites per muscle and the volume/dose injected into each muscle were determined at the discretion of the investigator [10-13].

In an early study by Poewe et al., aboBoNT/A was injected into only two muscles: SCM and SC [total doses of 250, 500, or 1,000 units divided among them and related to Toronto Western Spasmodic Torticollis Score (TWSTRS) result] [14].

Another early study, by Wissel et al., specified the range of doses per muscles of aboBoNT/A used: SCM: 100-200 units, SC 250-350 units, trapezius (Trap) 100-200 units and levator scapulae (LS) 100-200 units. The total maximum allowed dose per patient was 500 units. This reflects only the methodology of this trial, but does not show the actual dosages used per muscle in clinical practice [15].

In a more recent study by Poewe et al., the authors listed the injected muscles (LS, Trap, SCM, SC, scalene medius, semispinalis capitis and longissimus), but did not reveal the injected doses [16].
Figures regarding the dose per muscle in open label studies (more closely resembling our group of patients) are also scarce. Camargo et al. observed 28 patients with $\mathrm{CD}$ treated for seven years with BoNT/A and they reported doses consecutively injected into each muscle: SCM 15-75 units, Trap 30-100 units, SC 15-50 units, LS 15-50 units, and paravertebral muscles 15-50 units (doses were calculated for onaBoNT/A, but different preparations were used over the course of the seven years). This group was small $(\mathrm{n}=28)$ and doses were not assigned to a specific BoNT/A product for the whole follow up [2].

Bentivoglio et al. reported in a long term (at least six consecutive injections) open study of aboBoNT/A in CD the doses used for injected muscles: SCM, Trap, SM, SC, and LS. Mean dose (and SD plus range) for each muscle were: $110.0 \pm$ 44.9 (40-200) units for SCM, $231.4 \pm 158.3$ (60-500) units for Trap, $74.8 \pm 47.7$ (40-180) units for SM, $157.1 \pm 111.1(60-400)$ units for SC, and $118.7 \pm 57.9(60-300)$ units for LS. Nevertheless, the authors used the standard CD classification (as used in the pre-Col-Cap era). The most frequent dystonic patterns identified were torticollis and laterocollis, accounting for 
Table 2. Comparison between doses recommended in position statement of group of experts $(1,7)$ and mean doses used in our study (shown in bold; we selected more commonly used muscles)

\begin{tabular}{lccccc} 
Muscle & $\begin{array}{c}\text { ona/incoBoNT/A } \\
\text { (units, range) }\end{array}$ & $\begin{array}{c}\text { aboBoNT/A } \\
\text { (units, range) }\end{array}$ & $\begin{array}{c}\text { onaBoNT/A } \\
\text { (units, SD) }\end{array}$ & $\begin{array}{c}\text { incoBoNT/A } \\
\text { (units, SD) }\end{array}$ & $\begin{array}{c}\text { aboBoNT/A } \\
\text { (units, SD) }\end{array}$ \\
\hline Sternocleidomastoid & $20-50$ & $40-120$ & $40.8(15.5)$ & $34.7(15.2)$ & $117.9(40.1)$ \\
Splenius capitis & $40-100$ & $100-350$ & $49.2(26.0)$ & $36.8(21.2)$ & $139.6(80.7)$ \\
Trapezius & $25-100$ & $60-300$ & $29.2(13.2)$ & $33.0(13.3)$ & $123.4(47.7)$ \\
Levator scapulae & $20-100$ & $60-200$ & $32.8(12.3)$ & $39.0(17.9)$ & $135.8(50.8)$ \\
Semispinalis capitis & $20-100$ & $60-300$ & $19.7(13.2)$ & $30.7(15.2)$ & $111.4(63.4)$ \\
Splenius cervicis & $20-60$ & $60-140$ & $13.3(4.8)$ & $65.0(49.5)$ & $75.4(47.5)$ \\
Semispinalis cervicis & $20-60$ & $60-140$ & $18.0(8.5)$ & $31.3(14.3)$ & $102.1(73.9)$ \\
Obliquus capitis & $10-20$ & $60-200$ & $20.6(11.4)$ & $31.7(20.9)$ & $117.3(43.6)$
\end{tabular}

$78.7 \%$ and $78.5 \%$ of all the treatments respectively, followed by dystonic tremor (37.9\%) and shoulder elevation (14.4\%). Mixed patterns were a combination of torticollis and laterocollis (28.9\%) and torticollis with dystonic tremor (5.6\%) [1].

In our earlier publication regarding a cohort of 305 patients with $\mathrm{CD}$, we demonstrated that the most frequently injected muscle was the SC (83\%), followed by SCM (79.1\%) and Trap (58.5\%). But less frequently injected muscles were also treated: LS, semispinalis capitis (SScap), and OCI in $38.2 \%, 48.7 \%$ and $35.3 \%$ of patients respectively [17]. The most common pattern of CD was torticaput (49\%) followed by laterocaput (16.7\%). Pure forms were observed in $16.3 \%$ of patients only [5]. These 'new' muscles (i.e. those rarely included in clinical trials, such as $\mathrm{OCI}$ ) gained more awareness when the Col-Cap concept was introduced by Reichel at al. [6]. The doses chosen in our international cohort study are based on this relatively new approach. To the best of our knowledge, this has not been studied in such a large cohort previously.

The doses we used in our cohort are lower than in other studies with aboBoNT/A $[1,14,15]$. This may indirectly reflect the common use of US guidance in our practice which may result in more precise injections and may lower the dose per muscle. In all randomised and double-blind clinical studies published so far, US has not been used as the guidance technique [10-13]. The doses per muscle are lower for some muscles in our cohort compared to the recommendations of experts [4] - see Table 2.

The 'ideal' dose per muscle remains unknown because it has never been formally studied in a scientific manner. Nevertheless, the dose per muscle (after proper identification of dystonia pattern using the Col-Cap concept) could be crucial to effective treatment.

Our study, featuring a large cohort of 305 patients, reflects real life practice in movement disorder clinics using the same treatment regime across continents, and shows the doses identified as being 'effective' (all patients were treated previously with at least moderate/good response). It can potentially help as an overview for injectors going into the field of CD treatment.
We are aware that the use of ultrasound guidance is not yet available at some centres, and that others use other methods such as EMG guidance. Although this might influence the injection of some rare muscles, it should not significantly impair the fundamental results and implications of our study.

\section{Conclusions}

Our study shows, for the first time, the doses per muscle used in CD patients in real life practice using the Col-Cap concept, including muscles not previously injected.

The doses per muscle were lower than in other studies, which may be the result of both the Col-Cap concept and the use of (presumably more precise) US-guided injections.

\section{Funding: None.}

Conflicts of interest: Dr. Sanjay Pandey and Dr. Christian Altmann have no conflict of interest. All other authors were speakers and consultants for Allergan / Abbvie, Merz / Desitin and Ipsen, but none relevant to this study (and had no financial or other support).

Ethical approval: Depending on the national legal situation, this study was approved by the responsible ethics committees. Acknowledgment: The authors thank Tanja Fiesel who organised the data of the different centres.

\section{References}

1. Bentivoglio AR, Bagella CF, Albanese A. Botulinum toxin in movement disorders. In: Jankovic J, Tolosa E (eds) Parkinson's disease \& Movement Disorders 2015, 6th edn. Wolters Kluver, Philadelphia, pp.: 480-495.

2. Camargo $\mathrm{CH}$, Teive $\mathrm{HA}$, Becker $\mathrm{N}$, et al. Botulinum toxin type $\mathrm{A}$ and cervical dystonia: a seven-year follow-up. Arq Neuropsiquiatr. 2011; 69(5): 745-750, doi: 10.1590/s0004-282x2011000600004, indexed in Pubmed: 22042174.

3. Castelão M, Marques R, Duarte G, et al. Botulinum toxin type A therapy for cervical dystonia. Cochrane Database of Systematic Reviews. 2017, doi: 10.1002/14651858.cd003633.pub3. 
4. Albanese A, Abbruzzese G, Dressler D, et al. Practical guidance for $\mathrm{CD}$ management involving treatment of botulinum toxin: a consensus statement. J Neurol. 2015; 262(10): 2201-2213, doi: 10.1007/ s00415-015-7703-x, indexed in Pubmed: 25877834.

5. Jost WH, Tatu L, Pandey S, et al. Frequency of different subtypes of cervical dystonia: a prospective multicenter study according to ColCap concept. J Neural Transm (Vienna). 2020; 127(1): 45-50, doi: 10.1007/s00702-019-02116-7, indexed in Pubmed: 31828512.

6. Reichel G, Stenner A, Jahn A. The phenomenology of cervical dystonia Proposed New treatment strategy with botulinum toxin. Fortschr Neurol Psychiat. 2009; 77: 272-277.

7. Jost WH, Tatu L. Selection of Muscles for Botulinum Toxin Injections in Cervical Dystonia. Mov Disord Clin Pract. 2015; 2(3): 224-226, doi: 10.1002/mdc3.12172, indexed in Pubmed: 30838229.

8. Pandey S, Kreisler A, Drużdż A, et al. Tremor in Idiopathic Cervical Dystonia - Possible Implications for Botulinum Toxin Treatment Considering the Col-Cap Classification. Tremor Other Hyperkinet Mov (N Y). 2020; 10: 13, doi: 10.5334/tohm.63, indexed in Pubmed: 32775027.

9. Tyślerowicz M, Kiedrzyńska W, Adamkiewicz B, et al. Cervical dystonia - improving the effectiveness of botulinum toxin therapy. Neurol Neurochir Pol. 2020; 54(3): 232-242, doi: 10.5603/PJNNS.a2020.0021, indexed in Pubmed: 32285434.

10. Charles D, Brashear A, Hauser RA, et al. CD 140 Study Group. Efficacy, tolerability, and immunogenicity of onabotulinumtoxina in a randomized, double-blind, placebo-controlled trial for cervical dystonia. Clin Neuropharmacol. 2012; 35(5): 208-214, doi: 10.1097/ WNF.0b013e31826538c7, indexed in Pubmed: 22948497.

11. Comella C, Jankovic J, Truong D, et al. Efficacy and safety of incobotulinumtoxinA (NT 201, XEOMIN®, botulinum neurotoxin type A, without accessory proteins) in patients with cervical dystonia. Journal of the
Neurological Sciences. 2011; 308(1-2): 103-109, doi: 10.1016/j. jns.2011.05.041.

12. Truong D, Duane DD, Jankovic J, et al. Efficacy and safety of botulinum type A toxin (Dysport) in cervical dystonia: results of the first US randomized, double-blind, placebo-controlled study. Mov Disord. 2005; 20(7): 783-791, doi: 10.1002/mds.20403, indexed in Pubmed: 15736159.

13. Truong D, Brodsky M, Lew M, et al. Global Dysport Cervical Dystonia Study Group. Long-term efficacy and safety of botulinum toxin type A (Dysport) in cervical dystonia. Parkinsonism Relat Disord. 2010; 16(5): 316-323, doi: 10.1016/j.parkreldis.2010.03.002, indexed in Pubmed: 20359934.

14. Poewe W, Deuschl G, Nebe A, et al. What is the optimal dose of botulinum toxin $A$ in the treatment of cervical dystonia? Results of a double blind, placebo controlled, dose ranging study using Dysport. German Dystonia Study Group. J Neurol Neurosurg Psychiatry. 1998; 64(1): 13-17, doi: 10.1136/jnnp.64.1.13, indexed in Pubmed: 9436721.

15. Wissel J, Kanovsky P, Ruzicka E, et al. Efficacy and safety of a standardised 500 unit dose of Dysport in heterogeneous cervical dystonia population: result of a prospective, multicentre, randomised, doubleblind, placebo-controlled, parallel group study. J Neurol. 2001; 248: 1073-1078.

16. Poewe W, Burbaud P, Castelnovo G, et al. Efficacy and safety of abobotulinumtoxinA liquid formulation in cervical dystonia: A randomizedcontrolled trial. Mov Disord. 2016; 31(11): 1649-1657, doi: 10.1002/ mds.26760, indexed in Pubmed: 27653448.

17. Jost WH, Biering-Sørensen Bo, Drużdż A, et al. Preferred muscles in cervical dystonia. Neurol Neurochir Pol. 2020; 54(3): 277-279, doi: 10.5603/PJNNS.a2020.0022, indexed in Pubmed: 32227332. 